\title{
A Matched-Filter-Based Reverse-Time Migration Algorithm for Ground-Penetrating Radar Data
}

\author{
Carlton J. Leuschen, Student Member, IEEE, and Richard G. Plumb, Senior Member, IEEE
}

\begin{abstract}
Ground-penetrating radar (GPR) is a remote sensing technique used to obtain information on subsurface features from data collected over the surface. The process of collecting data may be viewed as mapping from the object space to an image space. Since most GPRs use broad beamwidth antennas, the energy reflected from a buried structure is recorded over a large lateral aperture in the image space. Migration algorithms are used to reconstruct an accurate scattering map by refocusing the recorded scattering events to their true spatial locations through a backpropagation process. The goal of this paper is to present a pair of finite-difference time-domain (FDTD) reverse-time migration algorithms for GPR data processing. Linear inverse scattering theory is used to develop a matched-filter response for the GPR problem. The reverse-time migration algorithms, developed for both bistatic and monostatic antenna configurations, are implemented via FDTD in the object space. Several examples are presented.
\end{abstract}

Index Terms-Finite-difference time domain (FDTD), ground penetrating radar (GPR), matched filter, reverse time migration, synthetic aperture radar (SAR).

\section{INTRODUCTION}

G ROUND-penetrating radar (GPR) is a mature remote sensing technique employed by engineers and scientists to obtain information from subsurface structures [1]. These structures range from manmade objects, such as buried utilities, pavements, and unexploded ordnance, to geological formations. The type of information extracted from a GPR depends in part on the manner in which data are collected. GPR data collection may be viewed as a mapping from the object space $(x, y, z)$, characterized by the object's spatial location and reflectivity, to the image space as shown in Fig. 1. The image space may be viewed in the space-time domain $(x, y, t)$, where the recorded scattered signals are displayed as a function of lateral position and time, or in the $\mathrm{F}-\mathrm{K}$ domain $\left(k_{x}, k_{y}, f\right)$, where the two image sets are related by spatial-temporal Fourier transforms (FTs). Additionally, data may be recorded in the space-frequency domain $(x, y, f)$, as would be the case with a frequency-domain GPR. Fourier transforms allow easy conversions between the three image domains.

Manuscript received April 28, 1998; revised June 17, 1999. This work was supported in part by NASA Graduate Student Research Fellowship NGT550073.

C. J. Leuschen is with the Radar Systems and Remote Sensing Laboratory, Department of Electrical Engineering and Computer Science, University of Kansas, Lawrence, KS 66044 USA.

R. G. Plumb was with the Radar Systems and Remote Sensing Laboratory, Department of Electrical Engineering and Computer Science, University of Kansas. He is now with the Department of Electrical Engineering, State University of New York at Binghamton, Binghamton, NY 13901 USA.

Publisher Item Identifier S 0196-2892(01)04016-5.

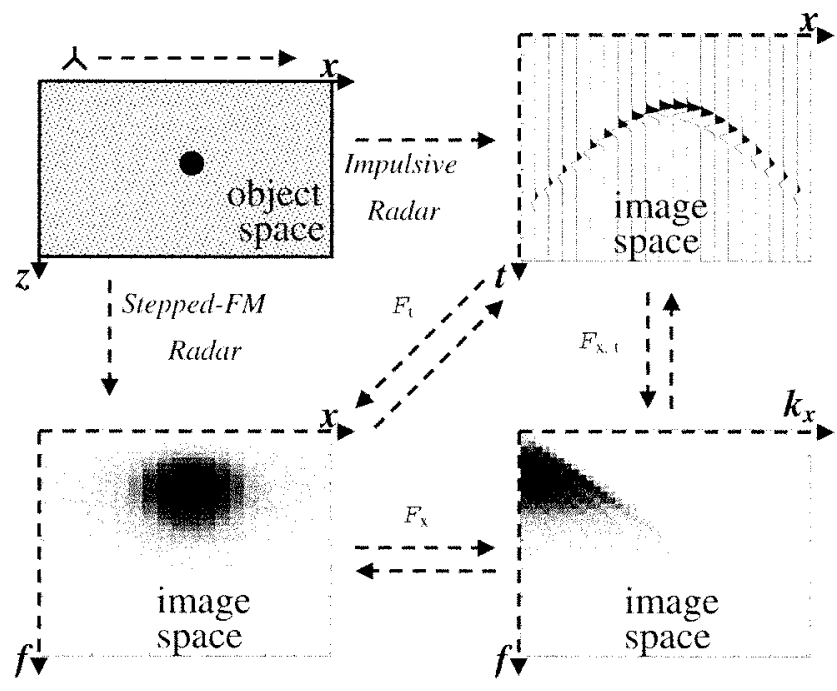

Fig. 1. Relationship between the object space and the three image spaces for GPR, where $\mathrm{F}_{t}$ and $\mathrm{F}_{x}$ denote temporal and spatial FTs, respectively.

Spatial location and reflectivity are the typical information obtained from GPR. Since most GPRs use broad beamwidth antennas, the energy reflected from a buried structure is recorded over a large lateral aperture in the image space. For example, in a monostatic survey, the data collected over a discrete object, such as a pipe, will appear as a diffraction hyperbola in the space-time image. No further processing may be needed if the goal is simply to detect the pipe. Imaging algorithms must be used, however, to move the observed scattering events to their true spatial location and to estimate the target's reflectivity.

Various imaging or migration techniques have been developed to refocus the scattered signals in the image space back to their true spatial location in the object space. Migration may be viewed as the inverse of data collection. It is the mapping from the image space to the object space [2], [3]. Some excellent reviews of the various migration techniques are presented in [3]-[5]. To summarize, migration algorithms may be implemented in the object space or in image space. Most algorithms are wave equation based, although a few are formulated on geometric considerations.

Many of the imaging algorithms used for GPR originated within the geophysical community and were developed with seismic applications in mind. Even though the wave excitation, scattering, and measurement associated with the practices of GPR and seismic applications are quite different, these techniques are frequently used because they often accomplish the initial goal of producing an image. The main scope of this paper is to present a comprehensive development of a generalized 
GPR imaging algorithm that is firmly based on electromagnetic theory and radar principles. By presenting this development in such a manner, we intend to give further understanding of the physical processes involved within GPR collection and imaging, present the assumptions necessary for the development and application of the algorithm and provide insight into the specific properties that are represented in an image.

The development of this algorithm is based on the notion of a matched filter, which is used extensively in radar applications. The matched-filter concept can be explained as a correlation of the received signal with the expected or estimated signal from a specific target. If this correlation produces a large value, then it is likely that the target is present. The implementation of a matched filter involves the estimation of this expected signal and an interpretation of the convolution. When electromagnetic scattering theory is used to determine the expected signal and the necessary assumptions are made, the resulting convolution takes on the form of a forward scattering problem. In fact, the resulting algorithm can be directly related to reverse-time migration (RTM) [6]-[10]. Using this development, an image can be perceived as a backpropagated wave-field reconstruction of the dielectric contrast within the ground.

In the following section, a matched-filter formulation for migrating GPR data for all source-receiver antenna configurations is developed using Born scattering theory for time-harmonic electromagnetic fields. A separate matched filter is then derived for the special case of the monostatic configuration. These matched filters are then implemented in the object space as FDTD reverse-time migration algorithms.

\section{MATCHED-FILTER FORMULATION}

In this section, a reverse-time migrating algorithm is developed using the concept of a matched filter. Mathematically, the matched-filter transfer function $H$ is expressed as the complex conjugate of the expected received waveform due to the target to which the filter is being matched. The output of the matched filter for $N$ transmitters, located at the position vectors $\mathbf{r}_{n}^{\prime \prime}$, and an $M$-element receiver array, located at the position vectors $\mathbf{r}_{m}$, is expressed as

$$
S\left(\mathbf{r}^{\prime}\right)=\sum_{n=1}^{N} \int \sum_{m=1}^{M} H\left(\mathbf{r}_{n}^{\prime \prime}, \mathbf{r}^{\prime}, \mathbf{r}_{m} ; \omega\right) \boldsymbol{U}_{\boldsymbol{n}}\left(\boldsymbol{r}_{\boldsymbol{m}} ; \omega\right) d \omega
$$

where $U_{n}$ is the received waveform due to the $n$th transmitter. In this formulation, the matched filter is taken directly from the expected received waveform, making it necessary to conjugate the collected data prior to filtering.

This section begins by estimating the time-harmonic electromagnetic fields scattered from an arbitrary object with a single receiver-transmitter configuration. This general result is then used to compute the expected scattering response from an isolated pixel containing one dominant point scatterer, which then leads to the matched filter for that pixel. In this development, the bistatic mode refers to any source-receiver antenna separation, while the monostatic mode indicates zero-offset data collection. Also, since a time-harmonic approach is taken, the frequency dependencies of the fields and signals have been omitted from the development and will be reintroduced later.

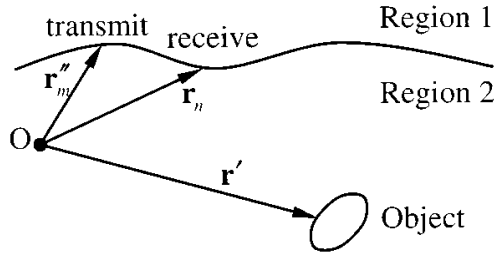

Fig. 2. Ground-penetrating radar (GPR) problem geometry.

\section{A. Electromagnetic Scattering}

Consider the problem geometry depicted in Fig. 2, consisting of two half spaces. Region 1 corresponds to free space while an inhomogeneous ground, characterized by constitutive parameters $\left\{\mu_{0}, \varepsilon_{2}(\mathrm{r})\right\}$, is denoted as Region 2. A weakly scattering object of finite size with constitutive parameters $\left\{\mu_{0}, \varepsilon(\mathbf{r})\right\}$ is located within the ground. Transmit and receive antennas are located at $\mathbf{r}_{n}^{\prime \prime}$ and $\mathbf{r}_{m}$, respectively.

Define a pair of wavenumbers with and without the scattering object present as

and

$$
k^{2}(\boldsymbol{r})= \begin{cases}\omega^{2} \mu_{0} \varepsilon_{0} & \mathbf{r} \in \text { Region } 1 \\ \omega^{2} \mu_{0} \varepsilon_{2} & \mathbf{r} \in \text { Region } 2 \\ \omega^{2} \mu_{0} \varepsilon & \mathbf{r} \in \text { Object }\end{cases}
$$

$$
k_{b}^{2}(\mathbf{r})= \begin{cases}\omega^{2} \mu_{0} \varepsilon_{0} & \boldsymbol{r} \in \text { Region } 1 \\ \omega^{2} \mu_{0} \varepsilon_{2} & \mathbf{r} \in \text { Region } 2\end{cases}
$$

respectively. By introducing an object profile as the difference between the dielectric constants in region 2

$$
O(\mathbf{r})=\varepsilon(\mathbf{r})-\varepsilon_{2}(\mathbf{r})
$$

and following the development in [11], [12], the time-harmonic scattered field at $\mathbf{r}$ due to an impressed source at $\mathbf{r}^{\prime \prime}$ may be expressed as

$$
\mathbf{E}_{s c a}(\mathbf{r})=-\int \overline{\mathbf{G}}\left(\mathbf{r}, \mathbf{r}^{\prime}\right)\left[k^{2}\left(\mathbf{r}^{\prime}\right)-k_{b}^{2}\left(\mathbf{r}^{\prime}\right)\right] \mathbf{E}\left(\mathbf{r}^{\prime}\right) d \mathbf{r}^{\prime}
$$

where $\overline{\mathbf{G}}\left(\mathbf{r}, \mathbf{r}^{\prime}\right)$ is the background dyadic Green's function that satisfies the equation

$$
\nabla \times \nabla \times \overline{\mathbf{G}}-k^{2} \overline{\mathbf{G}}=-\overline{\mathbf{I}} \delta
$$

with $\overline{\mathbf{I}}$ being the unit dyad and $\mathbf{E}\left(\mathbf{r}^{\prime}\right)$ being the total electric field inside the scattering object.

Since the total field inside the scatterer is a function of the object profile, (5) is a nonlinear integral equation. A Born approximation, which states that the total field inside the scatterer is approximately equal to the incident field, may be used to linearize (5), resulting in

$$
\mathbf{E}_{s c a}(\mathbf{r})=-\omega^{2} \mu_{0} \int \overline{\mathbf{G}}\left(\mathrm{r}, \mathbf{r}^{\prime}\right) O\left(\mathrm{r}^{\prime}\right) \mathbf{E}_{i n c}\left(\mathbf{r}^{\prime}\right) d \mathbf{r}^{\prime}
$$

where the incident field is expressed by the transmit signal $T$ and transmit antenna polarization, $\mathbf{u}_{t}$, as

$$
\mathbf{E}_{i n c}\left(\mathbf{r}^{\prime}\right)=j \omega \mu_{0} \overline{\mathbf{G}}\left(\mathbf{r}^{\prime}, \mathbf{r}^{\prime \prime}\right) T \mathbf{u}_{t} .
$$

The final migrated data may be viewed as an image consisting of a number of pixels. Assume that each pixel has one dominant 
point scatterer that can be modeled as an impulse in the object profile located at $\mathbf{r}_{s}^{\prime}$, the center of the pixel. The scattered field recorded at $\mathbf{r}$ due to this isolated point scatterer is

$$
\mathbf{E}_{s c a}(\mathbf{r})=-\omega^{2} \mu_{0} \overline{\mathbf{G}}\left(\mathbf{r}, \mathbf{r}^{\prime}\right) \mathbf{E}_{\text {inc }}\left(\mathbf{r}^{\prime}\right) .
$$

The received waveform, in volts, is related to the scattered field, in $\mathrm{V} / \mathrm{m}$, by the relationship [13]

$$
V_{o c}=\mathbf{h}^{*} \cdot \mathbf{E}_{s c a}
$$

where $\mathbf{h}$ is the antenna effective length in meters, and $V_{o c}$ is the open circuit voltage developed across the antenna terminals. If linear scattering is assumed (i.e., no interaction between scattering centers), then the matched filter for a point scatterer is taken directly from the received waveform by substituting (8) into (9) and projecting the scattered field onto $\mathbf{h}^{*}$

$$
H\left(\mathbf{r}, \mathbf{r}^{\prime}, \mathbf{r}^{\prime \prime}\right)=\mathbf{u}_{r}^{*}\left\{-\omega^{2} \mu_{0} \overline{\mathbf{G}}\left(\mathbf{r}, \mathbf{r}^{\prime}\right) j \omega \mu_{0} \overline{\mathbf{G}}\left(\mathbf{r}^{\prime}, \mathbf{r}^{\prime \prime}\right) T \mathbf{u}_{t}\right\}
$$

where $*$ denotes the complex conjugate, and $\mathbf{u}_{r}$ is the receive antenna effective length.

The final image is expressed by applying the complex conjugate of measured data to the filter

$$
S\left(\mathrm{r}^{\prime}\right)=\left\{j \omega \mu_{0} \overline{\mathbf{G}}\left(\mathrm{r}^{\prime}, \mathbf{r}\right)\left[-j \omega R \mathbf{u}_{r}\right]^{*}\right\}\left\{j \omega \mu_{0} \overline{\mathbf{G}}\left(\mathrm{r}^{\prime}, \mathrm{r}^{\prime \prime}\right) T \mathbf{u}_{t}\right\} .
$$

Through reciprocity, the arguments of the first Green's function have been reversed.

Equation (12) gives the migrated data as a function of frequency and the transmit and receive antenna locations. It is at this point that we take a step back from the mathematics of this expression and look at the physical interpretation. Equation (12) is divided into two values on the right-hand side. The first term is the electric field generated by a current source $\left[-j \omega R \mathbf{u}_{r}\right]^{*}$. If the time dependency of the received signal is introduced, this source is expressed as a derivative and time reversal $\left[-j \omega R \mathbf{u}_{r}\right]^{*} \Rightarrow R^{\prime}(-t) \mathbf{u}_{r}$. We will refer to the field generated by this source as the backpropagated electric field $\mathbf{E}_{b p}$. The second term is simply the incident field $\mathbf{E}_{i n c}$. Reintroducing the frequency dependencies and referring to (1), a complete expression for the migrated data is now shown as

$$
\begin{aligned}
& S\left(\mathbf{r}^{\prime}\right)=\sum_{n=1}^{N} \int \sum_{m=1}^{M} \mathbf{E}_{m n, b p}\left(\mathbf{r}^{\prime} ; \omega\right) \mathbf{E}_{n, i n c}\left(\mathbf{r}^{\prime} ; \omega\right) d \omega \\
& \mathbf{E}_{m n, b p}\left(\mathbf{r}^{\prime} ; \omega\right)=j \omega \mu_{0} \overline{\mathbf{G}}\left(\mathbf{r}_{m}, \mathbf{r}^{\prime} ; \omega\right)\left[-j \omega R_{n}(\omega) \mathbf{u}_{r}\right]^{*} \\
& \mathbf{E}_{n, i n c}\left(\mathbf{r}^{\prime} ; \omega\right)=j \omega \mu_{0} \overline{\mathbf{G}}\left(\mathbf{r}^{\prime}, \mathbf{r}_{n}^{\prime \prime} ; \omega\right) T(\omega) \mathbf{u}_{t}
\end{aligned}
$$

where the subscripts $m$ and $n$ denote the field or signal due to the $n$th transmitter and $m$ th receiver. These equations are now applied to bistatic and monostatic surveys.

\section{B. Implementation}

In the bistatic mode, data are collected from $N$ transmitter and $M$ receiver locations. Equation (13) indicates that $S\left(\mathbf{r}^{\prime}\right)$ is polarization dependent and therefore multivalued with respect to different combinations of $\left(\mathbf{u}_{r}, \mathbf{u}_{t}\right)$ and the definition of the pixel profile $O(\mathbf{r})$. A physical explanation of migrated data is obtained by recalling that the value of the pixel profile $O(\mathbf{r})$ was set equal to one in the previous section. Using this definition, the currents induced on the object by the incident field are $-j \omega O(\mathbf{r}) \mathbf{E}_{i n c}(\mathbf{r})$, while the scattered field is a direct result of these induced currents. Similarly, the collected data represent the currents induced on the receiving antenna by the scattered fields. In (14), these currents, associated with the $\left[-j \omega R \mathbf{u}_{r}\right]^{*}$ term, are reintroduced at the receive antenna locations and propagated through the object space via the same Green's function that described the propagation of the scattered field from the object to the receiving antenna during the collection process.

The final migrated data may then be represented in the time domain as the convolution of the incident electric field with the backpropagated electric field evaluated at time zero

$$
S\left(r^{\prime}\right)=\left.\sum_{n=1}^{N} \sum_{m=1}^{M} \mathbf{E}_{m n, b p}\left(\mathbf{r}^{\prime}\right) \otimes \mathbf{E}_{i n c}\left(\mathbf{r}^{\prime}\right)\right|_{t=0} .
$$

This expression is physically interpreted as the intersection of the backpropagated field with the incident field and consequently has strong ties to the reflector mapping formula used in seismic migration [14]. In fact, if the incident field in perceived to excite induced currents within the ground, this expression can be directly related to the excitation-time imaging condition [15].

The monostatic mode, or zero offset, is characterized by coincident transmit and receive locations and polarization, $\mathbf{r}=\mathbf{r}^{\prime}$ and $\mathbf{u}_{\mathbf{r}}=\mathbf{u}_{\mathbf{t}}$. From (12), the migrated data takes the form

$S\left(\mathbf{r}^{\prime}\right)=\left\{j \omega \mu_{0} \overline{\mathbf{G}}\left(\mathbf{r}^{\prime}, \mathbf{r}\right)\left[-j \omega R \mathbf{u}_{r}\right]^{*}\right\}\left\{j \omega \mu_{0} \overline{\mathbf{G}}\left(\mathbf{r}^{\prime}, \mathbf{r}\right) T \mathbf{u}_{r}\right\}$.

For the ideal case when the transmit waveform is an impulse $T(\mathbf{r}, t)=\delta(t),(17)$ is reduced to

$$
S\left(\mathbf{r}^{\prime}\right)=\left\{j \omega \mu_{0} \overline{\mathbf{G}}\left(\mathbf{r}^{\prime}, \mathbf{r}\right) \mathbf{u}_{r}\right\}^{2}[-j \omega R]^{*}
$$

which is in a form similar to (14).

At this point, zero-offset or monostatic migration may be represented using the exploding reflector model [16] as depicted in Fig. 3. The original problem has antennas located on the surface, which radiate an incident field into the ground. The incident field produces induced sources in the scatterer. These induced sources reradiate back toward the antennas along the same path as the incident field. In the exploding reflector model, induced sources are placed over the surface of the scatterer and are allowed to radiate upward at one half the speed of the original problem, or equivalently over twice the distance. By invoking reciprocity, these sources are moved to the surface and allowed to propagate back into the ground. The mathematical statement of the exploding reflector model when applied to electromagnetic propagation and scattering is

$$
\left\{j \omega \mu_{0} \overline{\mathbf{G}}\left(\mathbf{r}^{\prime}, \mathbf{r}\right) \mathbf{u}_{r}\right\}^{2}=j \omega \mu_{0} \overline{\mathbf{G}}\left(2 \mathbf{r}^{\prime}, 2 \mathbf{r}\right) \mathbf{u}_{r}
$$

where the squared term, representing the two-way propagation, has been replaced by a one-way propagation over twice the distance. 


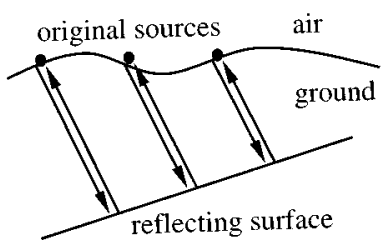

(a)

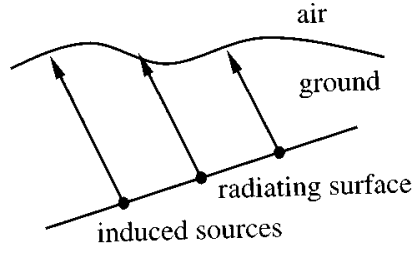

(b)
Fig. 3. Exploding reflector model.

Reintroducing the frequency dependencies and summing over the aperture results in

$$
\begin{aligned}
& S\left(\mathbf{r}^{\prime}\right)=\sum_{m=1}^{M} \int j \omega \mu_{0} \overline{\mathbf{G}}\left(2 \mathbf{r}^{\prime}, 2 \mathbf{r}_{m} ; \omega\right)\left[-j \omega R_{m}(\omega) \mathbf{u}_{r}\right]^{*} d \omega \\
& S\left(\mathbf{r}^{\prime}\right)=\sum_{m=1}^{M} \int \mathbf{E}_{m m, b p}\left(\mathbf{r}^{\prime} ; \omega\right) d \omega
\end{aligned}
$$

which is simply the back-propagated electric field in a space where all dimensions are increased by a factor of two. Finally, converting to the time domain yields

$$
S\left(\mathbf{r}^{\prime}\right)=\sum_{m=1}^{M} \mathbf{E}_{m m, b p}\left(\mathrm{r}^{\prime} ; t=0\right)
$$

which is related to the time-zero imaging condition [17]. Equation (22) is seen as an electromagnetic reverse-time migration algorithm and involves three basic steps: time reversing and differentiating the measured data, introducing these data as current sources at their receiver locations, and propagating the fields back into the ground until time zero. From here on, it is assumed that all spatial dimensions are increased by a factor of two for the monostatic configuration.

\section{IMPLEMENTATION VIA FDTD REVERSE-TIME MIGRATION}

In the previous section, matched filters for bistatic and monostatic migration were developed in the space-frequency image space. In this section we present a method of implementing migration in the object space via the finite-difference time-domain (FDTD) method. FDTD implementation offers several advantages: direct calculation of the half-space Green's function is avoided, any known inhomogeneities can be directly incorporated into the FDTD lattice, dispersive properties of the soil can be included, and multiple traces may be back propagated simultaneously. The need for corrections due to elevation changes is eliminated because the FDTD method allows the sources to be placed anywhere within the lattice. Also, the FDTD method provides a full vector solution of the wave equation. This eliminates the need to approximate (16) and (22) with scalar solutions and also allows for the inclusion of polarization measurements. The FDTD method was chosen due to the reasons stated above. However, the implementation of (16) and (22) are not limited by the FDTD method. Any forward-scattering technique is applicable to these equations and in some cases, another method may prove to be favorable.

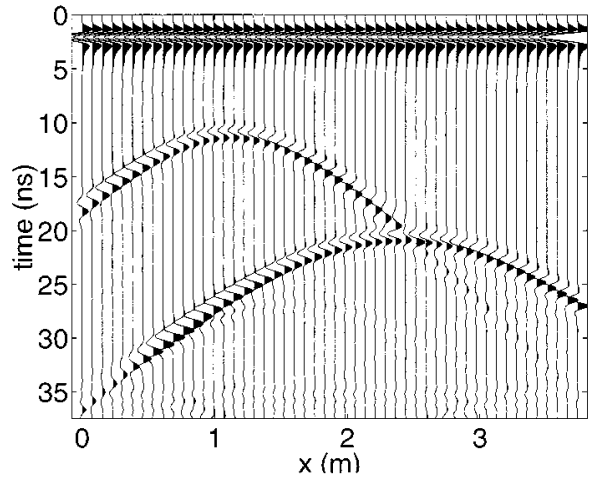

(a)

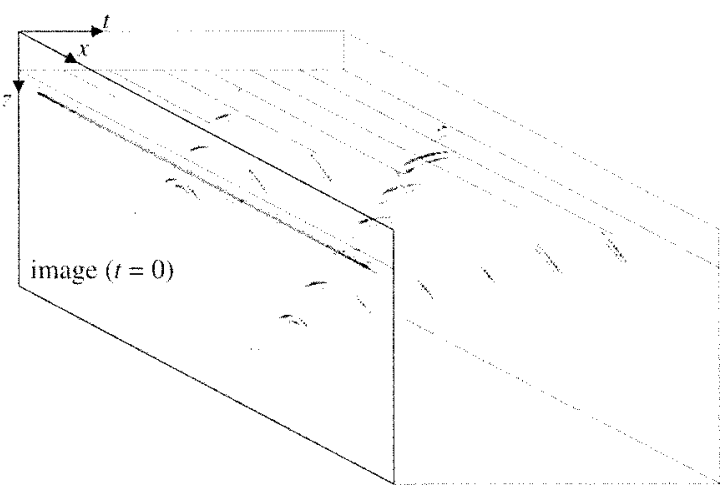

(b)

Fig. 4. Progression of the monostatic reverse-time migration algorithm. (a) Raw data from two localized targets are applied to the algorithm. (b) Illustration of the propagation of the wave where the image exists at time zero.

\section{A. Monostatic and Bistatic Implementation}

To implement (22) using FDTD, the $M$-measured traces, denoted as $R\left(r_{m}, t\right)$, are reversed in time $R\left(r_{m},-t\right)$, and the time derivatives are introduced into the object space as time-varying sources at the location where the individual traces were recorded. Using the time-reversed traces as spatialimpulsive sources, FDTD is then used to back propagate the recorded fields into the object space. The final migrated data, corresponding to a wavefront reconstruction, occurs at time $t=0$.

Fig. 4 illustrates the intermediate steps in the FDTD reverse-time migration process for monostatic data. Fig. 4(a) shows 50 traces of simulated data collected over a time window of $T=$ $37.5 \mathrm{~ns}$ in the presence of two localized targets. The locations of the individual targets are clearly seen at the apex of the two hyperbolas. Implementing the reverse-time migration begins by reversing and differentiating the datain time, introducing the time-reversed data as sources in the FDTD lattice at the individual receiver locations, and simultaneously exciting all 50 sources. Fig. 4(b) illustrates the progression of the backpropagated electric field with respect to time. The positive orientation of time in this figure is chosen to coincide with the forward scattering of the exploding reflector model, while the reverse-time migration is oriented in the negative time direction. Focusing begins to appear as the time approaches zero.

As discussed previously, the bistatic algorithm is implemented by convolving the incident field with the measured 


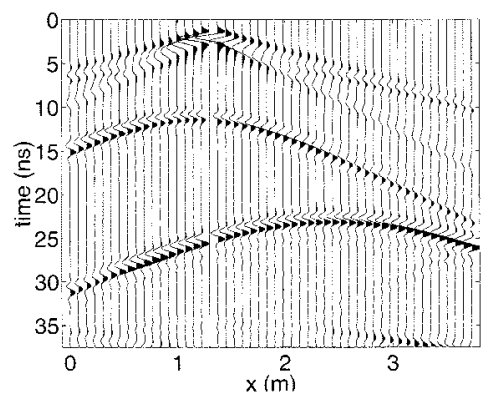

(a)
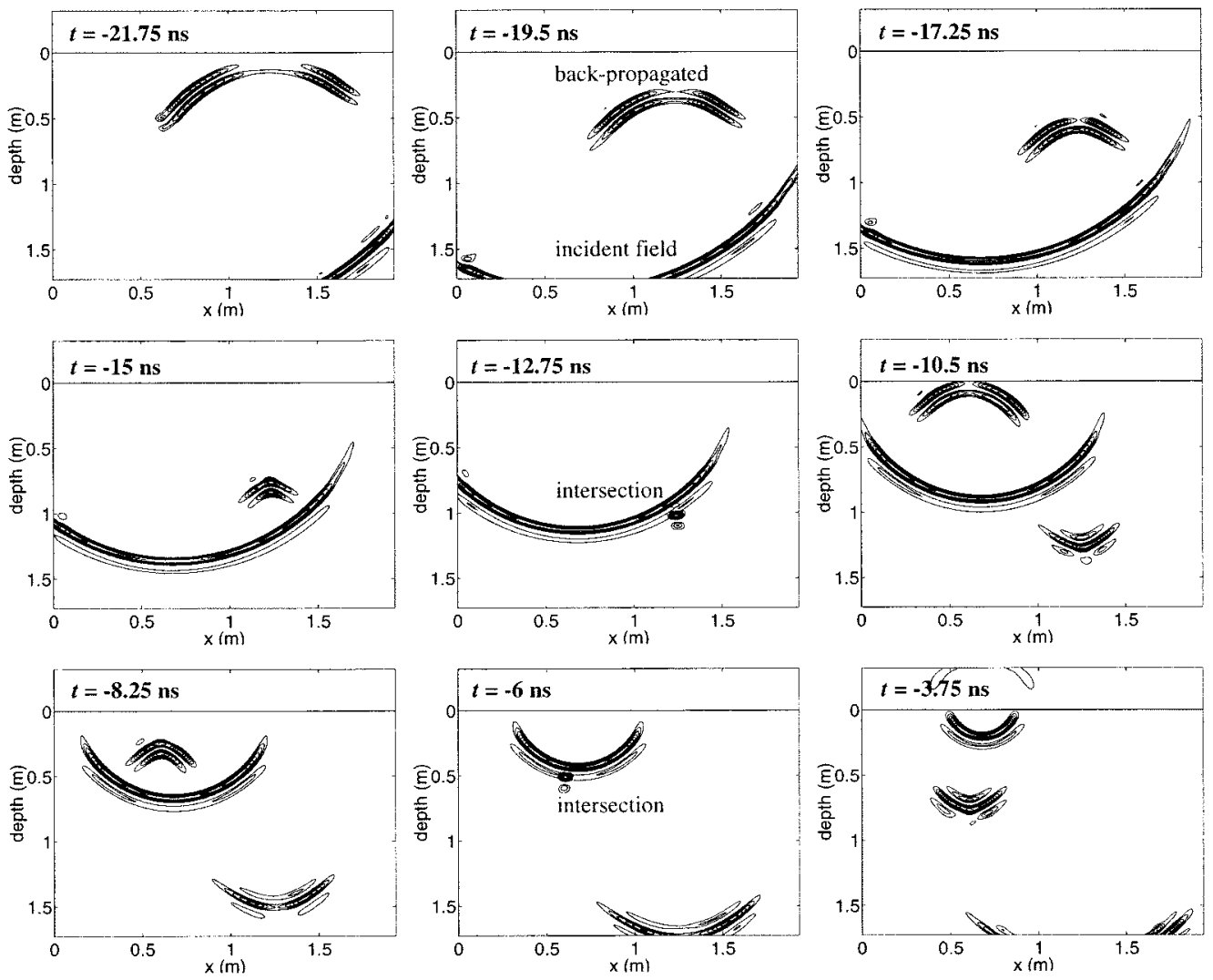

(b)

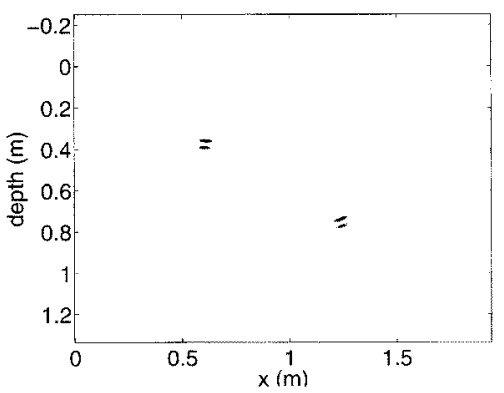

(c)

Fig. 5. Progression of bistatic reverse-time migration algorithm. The raw data (a) from two localized targets are applied to the algorithm. (b) Illustration of the incident and back-propagated fields with respect to time. (c) The final image is shown by the intersection of the two fields.

fields that have been backpropagated in time. There are several ways to implement this algorithm. One approach is to propagate each field individually while saving each field at every time step and then performing the convolution. This approach, although straightforward, requires an excessive amount of memory. The approach presented here is more efficient and is accomplished by propagating one of the fields in reverse while simultaneously propagating the other field forward. The image is expressed as a running sum of the product or intersection of the two fields as time progress. 
Specifically, the incident field is propagated forward in time while recording the fields for every time step at the absorbing boundaries and the entire field at the last time step.

The purpose of the absorbing boundaries is to eliminate any reflections at the boundaries of the lattice [18], [19]. This field can then be propagated in reverse by using a negative time step in FDTD and reintroducing the fields recorded at the boundaries merely as an alternative to the absorbing boundaries. As this incident field is propagated in reverse, the time-reversed recorded data are simultaneously propagated in another FDTD space by reintroducing the measured fields as sources at the receiver locations. As stated above, the convolution is accomplished by keeping a running sum of the product of the two fields.

Implementation of the bistatic algorithm is presented in Fig. 5. Here, bistatic data were simulated again for two localized objects. The raw data, collected at 49 receiver locations with the transmitter located directly above one of the two targets, are shown in Fig. 5(a). The bistatic algorithm requires propagation of both the incident and back-propagated fields. Fig. 5(b) shows the progression of the incident and backpropagated fields in time. Since the migrated data are obtained from the convolution of the incident field with the backpropagated field, contributions to the final image occur where the two fields intersect. For the present example this occurs at two locations, which are labeled in the figure. The result of the convolution is shown in Fig. 5(c).

\section{B. Experimental Results}

Data were collected in a $5 \mathrm{~m} \times 4 \mathrm{~m} \times 2 \mathrm{~m}$ deep, sand-filled, indoor GPR test facility at the University of Kansas, Lawrence. All measurements were collected using a Sensors and Software Pulse Ekko 1000 impulsive radar system with a bandwidth and center frequency of about $900 \mathrm{MHz}$. A sampling interval of $20 \mathrm{ps}$ was chosen to meet the stability criteria of the finite-difference time-domain method, while a total of 1500 samples were collected for each trace corresponding to a time interval of $30 \mathrm{~ns}$.

The first experimental configuration, depicted in Fig. 6, consisted of four 10-cm PVC pipes buried in dry sand. All the pipes were oriented along the $y$-axis simplifying the experiment into a two-dimensional (2-D) space of $x$ and $z$. The configuration shown in Fig. 6 indicates the relative locations of the PVC pipes, the concrete walls of the test facility, and 30 antenna positions. The three pipes at the surface were purposely separated by 20 and $30 \mathrm{~cm}$ center-to-center to investigate the lateral resolution of the algorithm, while the fourth pipe near the bottom is a permanent fixture of the box.

Data were recorded in a monostatic configuration. The individual traces, shown in Fig. 7(a), were collected with $y$-polarized antennas at each of the 30 locations. A linear gain was applied to the data only to bring out some of the deeper events in the figure, and it should be noted that this gain was not applied to the data prior to the match-filter processing. Once the monostatic data were recorded, implementing FDTD reverse-time migration was easily completed. The image shown in Fig. 7(b) is the result of the FDTD reverse-time migration along with a quadratic gain to account for spherical spreading.

Data were next recorded in a bistatic configuration. Due to the large number of transmitter and receiver combinations,

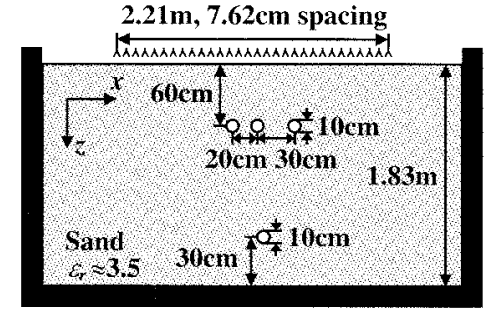

Fig. 6. Antenna and PVC pipe geometry for experiment number 1 .

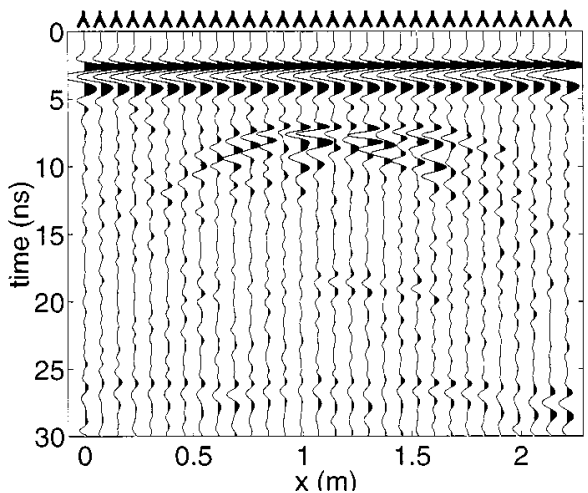

(a)

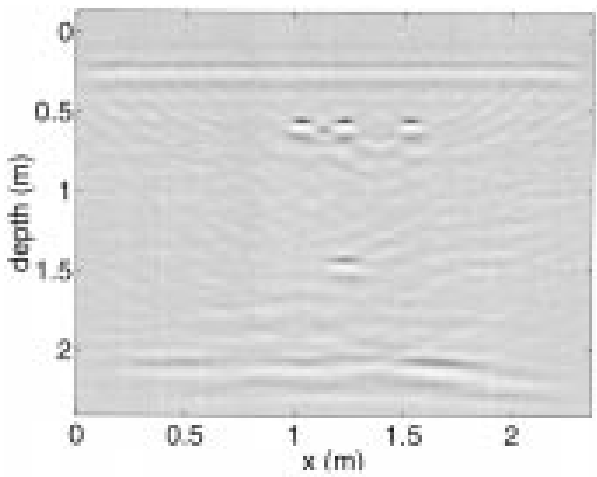

(b)

Fig. 7. Monostatic results for experiment number 1. (a) Raw data and (b) FDTD reverse-time migrated data.

a limited data set was collected. Seven common-shot data sets were recorded. Referring back to Fig. 6, location \#1 corresponds to the far right antenna location, while the numbers proceed to location \#30 on the far left. Using this configuration, the seven transmitter locations include \#2, \#6, \#10, \#14, \#18, \#22, and \#26. The traces shown in Fig. 8(a) were collected with the transmitter polarized in the $y$-direction at location \#6 with the receiver polarized in the $y$-direction at all 30 locations. The three traces near the transmitter were zeroed out since the receiving and transmitting antennas were in too close proximity. The image shown in Fig. 8(b) is a result of FDTD reverse-time migration of the recorded data convolved with the incident pulse. Again, a quadratic gain was applied due to spherical spreading. The same procedure was done on the bistatic data collected from location \#22. The image in Fig. 8(c) shows the sum of the images produce by both locations \#6 and \#22. Finally the data from all eight of the transmitter locations were combined to produce the image shown in Fig. 8(d). 


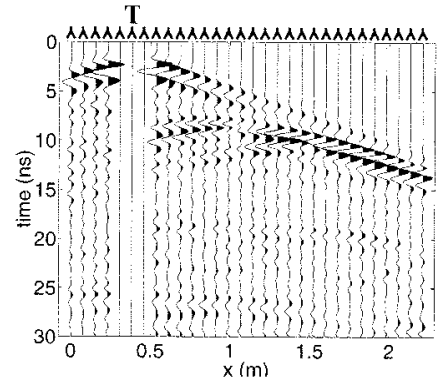

(a)

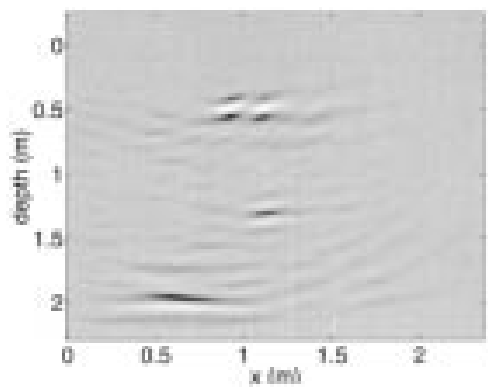

(b)

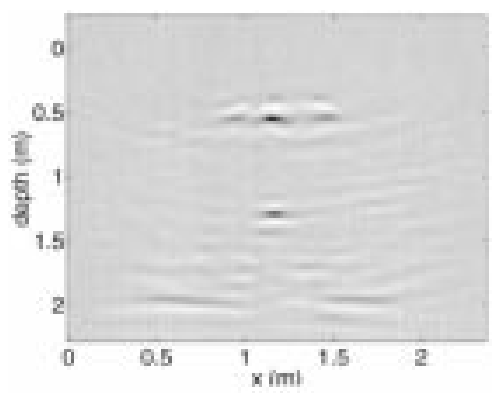

(c)

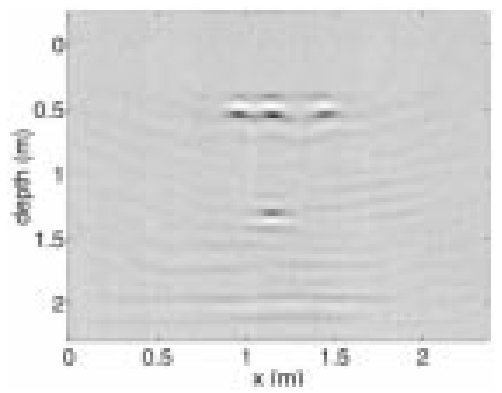

(d)

Fig. 8. Bistatic results for experiment number 1. (a) Common-shot raw data for the transmitter at location \#6. (b) FDTD reverse-time migrated data based on this one common-shot data set. (c) FDTD reverse-time migrated data using two common-shot data sets collected at transmitter locations \#6 and \#22. (d) FDTD reverse-time migrated data using all eight common-shot data sets.

The second experimental configuration consists of five 7.6 $\mathrm{cm}$ conduction spheres buried in an "L" shape at a depth of 60 $\mathrm{cm}$ as shown in Fig. 9(a). Measurements were collected similarly to the first configuration. However, since the objects were not homogeneous with respect to the $y$-axis, measurements were needed in both the $x$ - and $y$-directions on the surface. A total of 400 monostatic measures were collected to form a $7.6 \mathrm{~cm}$ grid over the surface. The migrated data corresponding to a constant depth of approximately $60 \mathrm{~cm}$ are shown in Fig. 9(b) and were generated using a three-dimensional (3-D) FDTD code.

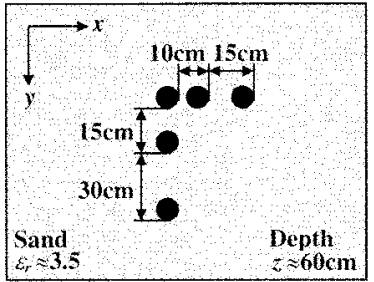

(a)

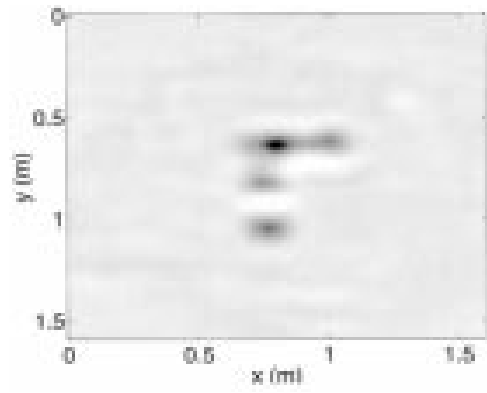

(b)

Fig. 9. Results for experiment number 2. (a) Problem geometry for experiment number 2 and (b) monostatic reverse-time migrated data.

\section{Discussion}

The problem of migrating GPR data has been investigated in this paper. Migration algorithms were first developed for processing seismic data sets and later brought over to process GPR data. The main goal of this paper was to develop a migration algorithm suitable for GPR applications. The motivation for this goal was to address two questions: what are the specific assumptions used when a migration algorithm is applied to GPR data, and what is the interpretation of the image generated by such an algorithm?

Using electromagnetic propagation and scattering theory along with the matched-filter definition, a migration algorithm was developed in a general sense [20] with a focus on reverse-time migration. The assumptions of this algorithm are stated as follows. First, the object profile is approximated by a set of point scatterers allowing a separate filter to be determined for each pixel. Next, each filter is made independent by assuming linear scattering. Finally, as with most imaging algorithms, some initial idea of the background media is necessary to propagate the fields. In addition, for the monostatic case, the assumptions inherent in the exploding reflector model apply.

From the above assumptions, the requirements for GPR wave migration include knowledge of the background media and weak scattering objects. The first requirement can usually be estimated by other means such as drilling cores or common midpoint surveys. However, the second requirement is rather vague and no definite limit can be expressed. For example, the 3-D experiment presented in Fig. 9 of this paper used conducting spheres, which are not weak scattering. Nevertheless, the correct results were obtained. For this case, as with many methods, the algorithm worked without completely satisfying all the assumptions. Thus, this second requirement provides a good rule of thumb, but the application of this algorithm can be expanded for most objects. 
The image generated by the reverse-time migration is a wavefront reconstruction. Since the algorithm is based on a matched filter, the image is expressed mathematically as a correlation of the received signal and the signal estimated in the presence of an object. The image is interpreted as an indication of the existence of the object, which in this case is the object profile or change in permittivity. As a result, the interpretation of an image is completely determined by the object profile. Using the notations used in the development, the image can now be defined as a wavefront reconstruction indicating a change in permittivity.

In this paper, GPR migration was viewed as a matched-filter process and implemented using FDTD. Implementing reverse-time migration algorithms with FDTD is effective. Because the recorded traces are treated as sources in the migration process, all traces or any subset may be backpropagated simultaneously. In addition, any a priori information may easily be incorporated in the FDTD lattice. For zero-offset or monostatic data, an image is obtained by superimposing waves obtained from backpropagating the measured data in the object space. For the bistatic case, the image is obtained by convolving the incident field with the backpropagated field. The examples presented in Fig. 8 illustrate the image quality improvement as a function of the number of common-transmitter surveys. As expected, image quality improves as the number of common-transmitter surveys increases. Although no examples were presented here, the bistatic algorithm is general enough to apply to nearly any transmitter receiver configuration.

\section{REFERENCES}

[1] J. D. Daniels, Surface-Penetrating Radar. London, U.K.: IEE, 1996.

[2] A. Tarantola, "Linearized inversion of seismic reflection data," Geophys. Prospect., vol. 32, pp. 998-1015, Dec. 1984.

[3] J. Gazdag and P. Sguazzero, "Migration of seismic data," Proc. IEEE, vol. 72, pp. 1302-1315, Oct. 1984

[4] A. J. Berkhout, "Wave field extrapolation techniques in seismic migration-A tutorial," Geophysics, vol. 46, pp. 1638-1656, 1981.

[5] - Seismic Migration-Imaging of Acoustic Energy by Wave Field Extrapolation-A. Theorectical Aspects. New York: Elsevier, 1982.

[6] E. Baysal, D. D. Kosloff, and J. W. C. Sherwood, "Reverse time migration," Geophysics, vol. 48, pp. 1514-1524, November 1983

[7] D. Loewenthal and I. R. Mufti, "Reversed time migration in spatial frequency domain," Geophysics, vol. 48, pp. 627-635, May 1983.

[8] W. F. Chang and G. A. McMechan, "3D reverse-time migration," Geophys. Prospect., vol. 37, no. 3, pp. 243-256, Apr. 1989.

[9] E. Fisher, G. A. McMechan, A. P. Annan, and S. W. Cosway, "Examples of reverse-time migration of single-channel, ground-penetrating radar profiles," Geophysics, vol. 57, pp. 577-586, Apr. 1992.

[10] J. Zhu and L. R. Lines, "Implicit interpolation in reverse-time migration," Geophysics, vol. 62, pp. 906-917, May-June 1997.

[11] W. C. Chew, Waves and Fields in Inhomogeneous Media. New York: Van Nostrand Reinhold, 1990.

[12] P. Chaturvedi and R. G. Plumb, "Electromagnetic imaging of underground targets using constrained optimization," IEEE Trans. Geosci. Remote Sensing, vol. 33, pp. 551-561, May 1995.
[13] W. L. Stutzman and G. A. Thiele, Antenna Theory and Design. New York: Wiley, 1981.

[14] J. F. Claerbout, "Toward a unified theory of reflector mapping," Geophysics, vol. 36, pp. 467-481, June 1971.

[15] W. F. Chang and G. A. McMechan, "Reverse-time migration of offset vertical seismic profiling data using the excitation-time imaging condition," Geophysics, vol. 51, pp. 67-84, 1986.

[16] D. Loewenthal, L. Lu, R. Roberson, and J. Sherwood, "The wave equation applied to migration," Geophys. Prospect., vol. 24, pp. 380-399, June 1976.

[17] G. A. McMechan, "Migration by extrapolation of time-dependent boundary values," Geophys. Prospect., vol. 31, pp. 413-420, June 1983.

[18] G. Mur, "Absorbing boundary conditions for the finite-difference approximation of the time-domain electromagnetic-field equations," IEEE Transactions on Electromagnetic Compatibility, vol. EMC-23, pp. 377-382, Nov. 1981.

[19] J. P. Berenger, "A perfectly matched layer for the absorption of electromagnetic waves," J. Comput Phys., vol. 114, pp. 185-200, 1994.

[20] C. Leuschen and R. G. Plumb, "A matched-filter approach to wave migration," J. Appl. Geophys., to be published.

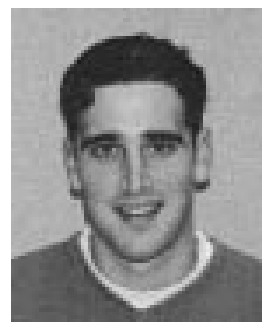

Carlton J. Leuschen (S'97) was born in Omaha, NE, on February 21, 1973. He received the B.S. and M.S. degrees in electrical engineering from the University of Kansas, Lawrence, in 1995 and 1997, with distinction and with honors, respectively. He is currently pursuing the $\mathrm{Ph} . \mathrm{D}$. in electrical engineering at the University of Kansas.

He has been with the Radar Systems and Remote Sensing Laboratory, University of Kansas, since 1995, where he has worked as a Graduate Teaching Assistant for the Sensor Design Laboratory. His research interests include GPR, numerical electromagnetics, and signal processing with emphasis on wave migration and SAR. In 1997, he was awarded a NASA Graduate Student Research Fellowship to develop a prototype GPR for Mars exploration.

Mr. Leuschen is a member of Tau Beta Pi.

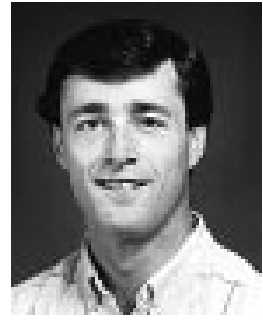

Richard G. Plumb (S'82-M'88-SM'94) was born in Syracuse, NY, in 1959. He received the B.S., M.S., and Ph.D. degrees in electrical engineering from Syracuse University in 1982, 1985, and 1988, respectively.

From 1982 to 1986 , he worked at General Electric, Syracuse. In 1985, he graduated from General Electric's Advanced Course in Engineering. Between 1986 and 1988, he was a Graduate Research Assistant with Syracuse University, and for the 1988 academic year, he was a Visiting Assistant Professor. In 1989, he joined the Faculty in the Department of Electrical Engineering and Computer Science, University of Kansas, Lawrence. In 1996, he was a Visiting Scholar at the University of Queensland, Brisbane, Australia. In 1998, he joined the Electrical Engineering Department, State University of New York, Binghamton, where he is currently Professor and Chair. His primary research interests are in electromagnetics, GPR, and inverse scattering. 